\title{
Mapping and size estimation of high-risk populations in large scale HIV prevention programs: how good is good enough?
}

\begin{abstract}
This paper assesses different approaches for mapping and size estimation used by implementing partners of Avahan: India AIDS Initiative, aiming to better understand each approach, its strengths and weaknesses, and the challenges in implementation among FSWs and MSM in the state of Maharashtra, Andhra Pradesh and Tamil Nadu, India. The different approaches used by Avahan partners identified through review of program documents, literature search and in-depth interviews were ethnographic mapping, broad mapping and site assessment, participatory site assessment, social network analysis, community-led situational needs assessment, mapping, enumeration and needs assessment and micro-planning. The seven approaches for mapping and size estimation, many of which used similar methods with varying degrees of community involvement and mapping was common to all, were used regularly to gather information over the first five years of implementation. The mapping and size estimation activities served multiple purposes and additionally to estimating the size and location information, information on risk and ways to intervene was also secured. Overall experience shows that routine updating of size estimates through mapping or other field-based exercises is critical to increase the coverage of high-risk populations in a given area and informs scaling up and real time assessment of coverage. Programmatically, mapping and size estimation exercises cannot be limited to 'just' counting the numbers; there is a need to collect and assimilate information on the risk behaviors, mobility of the target population and its vulnerability. Incorporating routine size estimation activities into national programming is essential as this will improve programs ability to assess coverage and thereby identify gaps and inform effective scaling up over time.
\end{abstract}

Keywords: mapping, size estimation, high-risk population, avahan, FSW, MSM, HIV/STI, social network analysis, class
Volume 5 Issue 5 - 2017

\author{
Shreena Ramanathan,' Prabuddhagopal \\ Goswami, ${ }^{2}$ Bitra George, ${ }^{2}$ Lakshmi \\ Ramakrishnan,' Rajatashuvra Adhikary,' \\ Sanjeev S Gaikwad,' R Ramakrishna, ${ }^{3}$ Rajesh \\ Kumar Patra, ${ }^{4}$ S Radhakrishnan, ${ }^{5}$ Lakshmi \\ Bai, ${ }^{6}$ Philip Kumar ${ }^{7}$ \\ 'Formerly with FHI 360, India \\ ${ }^{2} \mathrm{FHI} 360$, India \\ ${ }^{3}$ India HIV/AIDS Alliance, India \\ ${ }^{4}$ Formerly with HLFPPT, India \\ ${ }^{5}$ Formerly with Pathfinder International, India \\ ${ }^{6} \mathrm{TAI}, \mathrm{Tamil} \mathrm{Nadu}$, India \\ ${ }^{7}$ Independent consultant, India
}

Correspondence: Shreena Ramanathan, Formerly with FHI 360, India, India, Tel 919582570523, Email bgeorge@fhi360.org

Received: November 01, 2016 | Published: April 17, 2017
Abbreviations: HIV, human immunodeficiency virus; AIDS, acquired immune deficiency syndrome; FSW, female sex workers; MSM, men who have sex with men; TG, transgender; IDU, injecting drug user; STI, sexually transmitted infections; NGO, non-governmental organization; HRG, high risk group; TIL, targeted interventions; PSA, participatory site assessment; SNA, social network analysis; CLASS, community-led situational needs assessment; MENA, mapping enumeration and needs assessment.

\section{Introduction}

Determining the size of most-at-risk population groups is crucial for planning and implementing HIV/STI prevention programs in concentrated epidemics, where injecting drug users, men who have sex with men, and female sex workers and their clients need to be prioritized for intervention. Program implementers have multiple purposes for data collection, including (a) estimating the size of target populations, (b) defining locations of high-risk populations for interventions, and (c) securing data on risk behaviors and perceptions, among others, to inform interventions. Reliable information about the size of high-risk population in a given geography forms the basis for funding, setting performance targets, allocating program resources, and initiating policy change. ${ }^{1}$ Estimating the size of populations with high-risk sexual behavior is a challenging task due to the difficulty in accessing these groups. Different methods of mapping and size estimation are used globally, including census, enumeration, population surveys, capture-recapture method,,$^{2-4}$ multiplier method, ${ }^{5,6}$ and network scale up method. ${ }^{7}$ However, there are no gold standards or a single method that is recommended universally to estimate the size of the risk population. ${ }^{8}$ Application of direct methods, such as census and enumeration, is neither feasible nor cost-effective for hidden and hard-to-reach populations. Indirect methods, such as capture-recapture and multiplier methods, require development of a sampling frame and data from multiple sources, which is often difficult to obtain or unavailable. ${ }^{9,10}$

\section{Program description}

Avahan: India AIDS Initiative is one of the largest HIV prevention programs in India. Aiming to arrest the spread of HIV, the program was initiated in 2003 across six Indian states reporting the highest HIV prevalence in 2002. The six states are Tamil Nadu, Karnataka, Andhra Pradesh, Maharashtra, Nagaland and Manipur, which together accounted for 83percent of the HIV infections in India. ${ }^{11}$ The program targeted populations with high-risk sexual behaviors, including female sex workers (FSWs) and their clients, high-risk men who have sex with men (HR-MSM), transgender population, injecting drug users (IDUs), and long distance truck drivers. ${ }^{12}$ Avahan provided a 
package of proven prevention interventions, such as peer-led outreach to promote behavioral change, clinical services to treat STIs, social marketing and distribution of free condoms, support for community mobilization and advocacy to reduce structural barrier to safer sexual practices. By December 2008, Avahan's peer educators had reached 350,000 FSWs and 100,000 HR-MSM. ${ }^{13-15}$ Targets for the program were determined through regular mapping, size estimation exercises and micro-planning processes that form the basis of this paper. The program is currently in its second phase and being transitioned to its natural owners: Government of India and the local communities. ${ }^{15}$

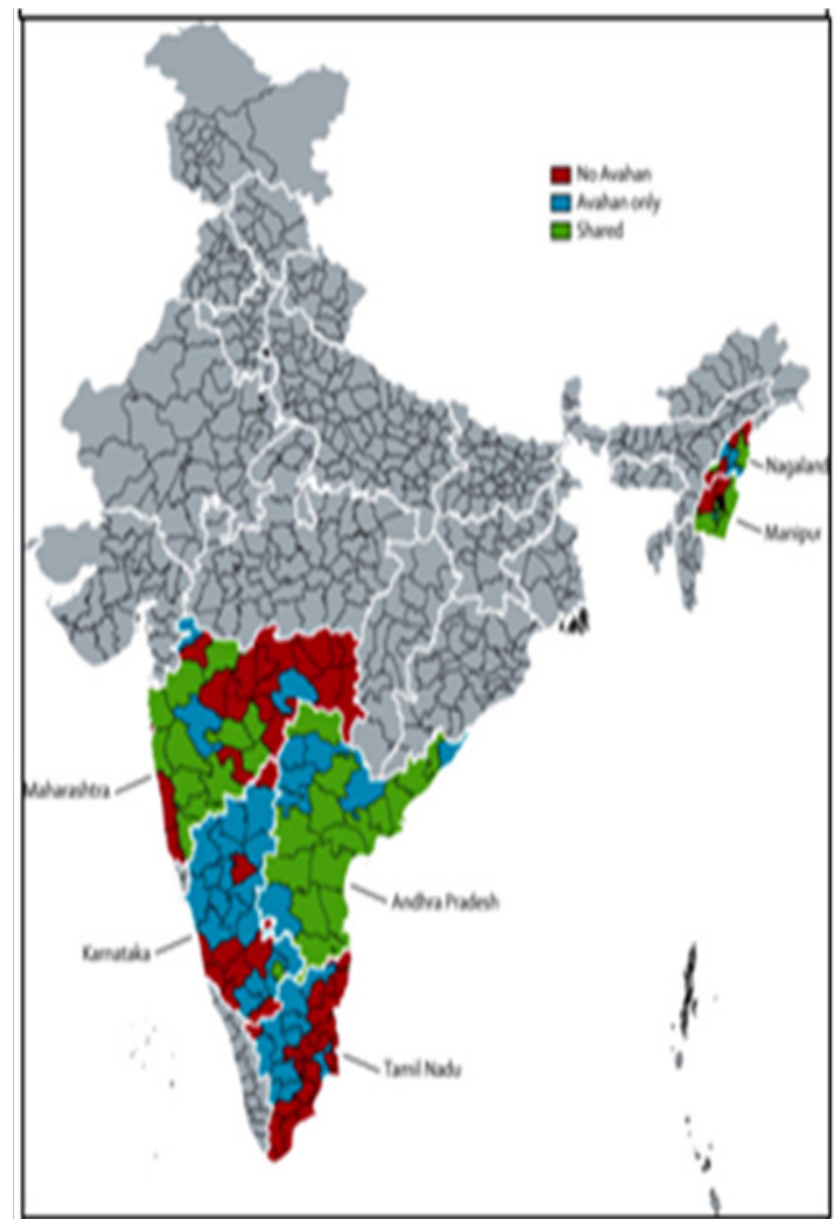

Figure I Avahan's reach Map showing states and districts where Avahan program was implemented.

\section{Materials and methods}

\section{Assessment of approaches for mapping and size estimation}

We focus here on the approaches for mapping and size estimation used by Avahan's implementing partners among FSWs and HRMSM in the three Indian states of Andhra Pradesh, Tamil Nadu and Maharashtra. In the current analysis, the mapping and size estimation approaches were assessed on several aspects, including: (a) Operational definitions; (b) Types of information gathered; (c) Level of involvement of members of high-risk populations; (d) Methods of validation and triangulation; (e) Frequency of mapping and size estimation activities Different sources were used to gather information about the approaches used in Avahan. Firstly, mapping and size estimation reports (both published and unpublished) were gathered from implementation partners in the three states, two each in Andhra Pradesh and Maharashtra and one in Tamil Nadu. Secondly, a web search was undertaken to find peer reviewed publications on mapping and size estimation approaches elsewhere. In-depth interviews were carried out with staff of implementation agencies involved in mapping and size estimation. In all we conducted 12 interviews during March-July 2011, with two to three program staff in each of the three states. The staffs were selected for the interview based on their association with mapping and size estimation activities. Permissions for interviews were obtained from Project Directors, and verbal consent was taken from the respondents. Interview records were transcribed and coded according to different themes and issues.

\section{Result}

\section{Mapping and size estimation approaches used in Avahan interventions}

The partners mentioned seven approaches and described their perceived strengths and weaknesses for mapping and size estimation, many of which used similar methods with varying degrees of community involvement, key informant interviews, involving creation of site maps and estimating site specific sizes. Adaptations of the general approach were used by the various state programs and are detailed in Table 1.

\section{Challenges in mapping and size estimation of high-risk groups}

Rigorous evidence-based approaches are difficult to devise in field situations by intervention programs, unlike in research studies. Our assessment of the different approaches of mapping and size estimation showed that program implementers did not use a uniform operational definition for the target population. The operational definitions used were based on practical issues useful to programming and resource allocation. While age criterion was used, no specific time frame such as past month or six months was incorporated in the operational definition for FSWs. Given the practical considerations and the program's aim to reach all high-risk population members, it was important to have such flexibility. From the analytical perspective, however, this lack of a common operational definition made it challenging to aggregate data and make direct comparisons with other data sources. Mapping and size estimation exercises were not conducted at regular intervals, and the frequency varied from place to place across geographic locations. Some implementing partners repeated them on an annual basis, while others did them less frequently (once in two years). The implementing partners who conducted these exercises less frequently mentioned that the data was updated regularly, based on the routine program monitoring data. Another reason for infrequent mapping and size estimation was that the numbers estimated at the initial stage of the program were large, and saturated coverage could not be achieved. Some criteria used for repeating mapping and size estimation, included trends in the routine program monitoring data with regard to the number of monthly contacts, dropouts from the program, and the mobility pattern of the population in a given area. Since uniform criteria were not adopted for routinely updating site-specific population size, comparisons across programs and geographies proved difficult. 
Table I Descriptions of mapping and size estimation methods used in Maharashtra, Andhra Pradesh and Tamil Nadu\#

\section{Approach name: Ethnographic mapping \\ State(s): Tamil Nadu (2003-2007), Andhra Pradesh \\ Population covered: FSWs \\ General description: ${ }^{16,17}$}

Broader methodological steps involve dividing each target area into smaller geographical units and collecting data within each unit from secondary (pimps, agents, brokers, madams, hotel workers) and tertiary key informants (NGO workers, government officials, pharmacy owners). Information is also gathered through participan observation of the population's geographical distribution in a given area, the typologies and the dynamics of the population for design of prevention interventions. The next step involves validation of estimates and other information by engaging communities in identifying and mapping out details related to hotspots, locations, numbers, days, and time, followed by triangulation of final results.

\section{Strengths}

- The resulting estimates may be more reliable, given the involvement of and validation by high-risk population members

- This method is used for planning interventions, as it provides size estimate, locations of hotspots and established contact with communities

- It can be utilized early in the program, when relationship is established with only a few members of the target population

Limitations

- The method may prove time consuming and costly

- It needs field staff that are familiar with the approach and acceptable to the target population

Approach name: Participatory site assessment (PSA) State(s): Andhra Pradesh (2002-2008), Maharashtra (2006) Population covered: FSWs, MSM General description: ${ }^{19-21}$

The PSA process was done in two discrete stages, with different objectives and two separate sets of participatory research tools. The first stage, PSAI, concentrated on identifying the neighborhoo and areas with concentrations of sex workers, estimating the numbers of different categories of sex workers, and finding out the peak and low 'business' times of sex workers, among others. The second stage, PSA2, involved more in-depth explorations to understand the specific factors that make sex workers particularly vulnerable to HIV and AIDS, the ways in which social marginalization and stigma against sex work exacerbate their vulnerability, and the profile of clients who engage their professional services. PSA2 also mapped existing services, both specific to HIVIAIDS and to other health, education and social development issues, along with recording sex workers' perceptions about service quality. PSA2 also explored local sex workers' ideas about how the project should be implemented at site to maximize its utilization and benefits for sex workers. Trained members of the high-risk population carried out PSA. At the end of every phase, the PSA teams come togethe for collective feedback workshops. These workshops collated information from sites and discussed strategies that worked well or otherwise, enabling teams to make comparisons across sites and facilitate cross learning.

\section{Strengths}

- As a method PSA is both an assessment process as well as an intervention. It not only gathers information about the high-risk population but also more explicitly mobilizes and builds capacity of the high-risk community members, who may, at a later stage, be used for implementing HIV prevention programs in the area

\section{Limitations}

- As PSA demands a significant commitment of time from community members, it becomes essential to plan for a higher dropout rate of trained community members and to assess their willingness to travel to other districts.
Approach name: Broad mapping and site assessment State(s): Tamil Nadu,Andhra Pradesh, Maharashtra (2008 onwards) Population covered: FSWs, HR-MSM

General description: ${ }^{18}$

The process of broad mapping and site assessment involves three steps: (I) review of secondary data, (2) broad mapping to estimate size and identify high-risk group (HRG) typology and locations of risk, and (3) site assessment to derive basic insights into factors that make HRGs particularly vulnerable to HIV. Review of secondary data is undertaken at the state level to consolidate information from existing targeted interventions (Tls) and to identify areas where known concentrations of high-risk populations exist but there are no Tls. Broad mapping is undertaken by peer educators/outreach workers with the support of high risk community members at site level to generate a list of hotspots, estimate size of high-risk populations by sub-categories and map the available HIV/STI services. Site assessment is carried out within a site at the hotspot to assess and confirm the list of hotspots, estimated size, availability, and mobility pattern of highrisk populations.

Strengths

This approach is useful in designing and planning intervention programs

It can be carried out in a shorter time period than ethnographic

mapping. The size estimate it yields can be used as a denominator at the start of a program

It is useful after the initial work with the community has started and trust gained

Limitations

Compared to ethnographic mapping, the approach requires greater participation of members of the community being mapped

Approach name: Social network analysis (SNA)

State(s): Andhra Pradesh (2006-2010)

Population covered: FSWs, MSM

General description: ${ }^{2 !}$

SNA is an effective tool for understanding the various networks that exist at the hotspot level. These networks can be used for effective, need-based outreach because categorization is done in terms of low, medium and high-risk class. These classifications are based on indicators such as condom use, number and kinds of sexual acts and client profiles. The SNA approach used for the program had a participatory methodology that involved community members. Two social science research techniques were used in SNA - mapping and in-depth interviews. With the help of existing social networks of community members (FSWs, HR-MSMs/TGs), detailed and accurate information was collected to revalidate enumerated numbers. Community participation was ensured in planning and implementation process, designing the questionnaire, and training the selected members on the tool, fieldwork, and analysis and drawing conclusions for each intervention. It helped the team understand community members' risk behavior, vulnerability to infection, methods of prevention, and, most importantly, the connection/relation with each member within the community.

Strengths

SNA goes beyond PSA and size estimation and lists out information pertaining to the high-risk populations' socio-economic status, risk behavior and networks

It is helpful in generating an exhaustive list of high-risk populations and their risk profile, which can be compared with program data Limitations

As data collection requires good rapport with the community, SNA is not an appropriate method for size estimation or mapping, especially in the initial stages of program implementation. 
Table Continued..

Approach name: Community-led situational needs assessment (CLASS)

State(s): Andhra Pradesh (2005)

Population covered: HR-MSM

General description: ${ }^{21,22}$

CLASS is a participatory approach adapted from the published

PSA approaches, involving members from the target population in planning and implementation of the program. The CLASS process was undertaken in two stages-CLASS I and CLASS 2. Each stage hade different objectives and made use of a different set of participatory research tools. The rationale for conducting CLASS in two distinct stages was twofold. Firstly, the participatory research tools used in CLASSI were relatively simple and easier to implement by firsttime researchers. By dividing the entire process into two stages, key populations (KPs) got the opportunity to gain confidence as well as the necessary skills to administer the relatively complex and in-depth tools of the second stage. Secondly, by going back to the same area, the KP researchers were able to earn the trust of $\mathrm{KPs}$ in the field; consequently, the latter were more forthcoming in sharing the sensitive information sought in CLASS 2.The first stage of CLASSI focused on: identifying the neighborhoods and areas in the intervention sites where there was concentration of HR-MSM; estimating the numbers of MSM of different categories in these areasThis method is dependent on the information provided by key or hotspots; and finding out the times when MSM were available at informants and sex workers contacted in the initial stages of the the hotspots and their mobility patterns. The second stage of CLASS program. Certain typologies may get missed, depending on the 2 involved more in-depth explorations to understand the specific knowledge of the initial informants factors that made MSM particularly vulnerable to HIVIAIDS and the ways in which social marginalization and stigma against MSM exacerbated their vulnerability. The tools used in this stage provided information on the profile of clients who engaged their services. This was followed by dissemination within the community and feedback sessions.

Strengths

The conduct of this exercise in a phased manner allows the team to earn the community's trust and establish rapport

It involves the target population in planning and implementation stages of the program

If successful, jump starts program implementation

Limitations

To be properly implemented, this approach requires full cooperation of the community

Approach name: Micro-planning

State(s) used: Maharashtra (2004-2008)

Population covered: FSWs

General description: $:^{13,23,24}$

Micro-planning is a system peer educators use during outreach to record and analyze risk. Micro-planning tailor's services to those at highest risk and least served and start building management and leadership skills in the high-risk community. Outreach planning is crucial to micro-panning and constitutes local level mapping, which takes place in two stages. First, peers draw street maps of hotspots with pictorial depiction of sex solicitation venues (such as bus stations, public toilets, parks) and significant buildings (such as hospitals, police station, shops). The number and locations of high-risk individuals are also marked on maps. This local level mapping is repeated every six months. In the second stage, peers create maps of social networks. These non-topographical maps list the names of friends and acquaintances in their community and use symbols and colors to mark typologies, risk characteristics and connections. These maps are useful in planning outreach. Specific micro-planning tools were developed to assess risks and vulnerabilities. Two tracking forms were commonly used: a daily tracking form and a monthly tracking form. The daily tracking form was designed for use by low-literate or illiterate peers, who could complete the form with stickers and symbols rather than writing. The monthly form, also known as a micro-planning calendar, aggregated data from peer interactions and was updated at least once a week.

Strengths

Micro-planning allows tracking and monitoring of individual members of high-risk groups

It provides peers an understanding of the changing risk and vulnerability of the individuals they serve

This approach improves the program's service delivery levels

It incurs no additional cost

Limitations

One of the limitations of micro-planning is that it largely depends on the reach of peer educators, and supportive supervision is required to ensure that sex workers are not missed out

Being an internal process, it necessitates some kind of external validation mechanisms to ensure accuracy 
Involving community members in mapping and size estimation during fieldwork was important, as they were aware of both the context as well as the geographic area. However, during the initial stages of the program, community members were often reluctant to associate with the program and its activities; resistance also came from agents, pimps, middlemen and brothel owners. Some programs tackled these issues by explicitly involving members in community building activities before undertaking more formal mapping and size estimates; these initiatives sought to reduce reluctance among leaders and allowed them to participate fully in field-based activities. It was considered important to create a physical and social space for the members of the high-risk groups to get together, bond, discuss problems and possible solutions, and build their own capacities to influence their environment. These efforts sought to ensure greater involvement of the high-risk population in the program and yield better size estimates. However, involvement of the target population also posed certain challenges, like high turnover. More than the required number of community members had to be trained to offset the periodic staff dropouts. The level of community involvement in population mapping and size estimation also varied across approaches. In some cases, more than half of the team that carried out mapping and size estimation came from the community, while in others their participation was as low as 10-20percent. Community participation also depended on the maturity of the program and the trust the community placed on an implementing agency. Triangulation of the data gathered from different sources was an important step in most approaches. However, at times it was difficult to reach a consensus on the numbers in a given location. The many ways in which the numbers, locations mapped, and other information gathered was authenticated included workshops with community members, key informants and other stakeholders and back checking and repeating the exercise (done on 10percent of the sites using a second set of field workers after data collection). In some cases, qualitative methods like focus group discussion and mystery client techniques were used to validate the estimated size of population. The other external factors that impinged on the mapping and size estimation exercises were police raids, festivals, mobility, and the local dynamics of the communities.

\section{Discussion}

This paper reviews field-based approaches for mapping and size estimation of high-risk populations during implementation of a large-scale HIV prevention program across India. The approaches used by Avahan: India AIDS Initiative provided insights that are critical for program planning and illustrate how estimates of size and location and information on risk profile and availability of services are collected. An important learning from the current review is that during implementation of different approaches for data collection to meet multiple/complex programmatic needs, it is essential to ensure flexibility and responsiveness to unique needs of geographic areas, social context and other characteristics of high-risk populations. The findings reveals that the program incorporated mapping and size estimation as a routine program activity and used the data in an ongoing manner to assess and improve program coverage over time. The experience from Avahan program has been that routine updating of size estimates through mapping or other field-based exercises is a critical component in achieving high coverage of high-risk groups in a given area. All the implementing partners under review provided services across vast geographies, often across a district and/or state. Large scale by design, the program had the objective to reach saturated coverage-80percent or more of the estimated target group in a geographic area. Hence, the activities informing such as a program, including mapping and size estimation exercises, are important to ensure effective coverage of a large number of areas and multiple geographies. The program thus needed realistic and heterogeneous approaches that helped it have a good handle on the population, instead of trying to apply one method or getting precise size estimates. It therefore employed different approaches to generate the information needed for programming-realistic denominator estimates, location and size of hotspots, and additional risk information to tailor interventions. Several challenges were faced in applying different mapping and size estimation methods, including ensuring active participation of high-risk populations, reaching consensus on size, and community members dropping out from field teams during estimation activities. These challenges could, however, be addressed, and the important learning to emerge from the current analysis is that approaches such as participatory site assessment and social network analysis not only provide size estimates and hotspot locations but also prove helpful in risk profiling and developing capacities and a sense of ownership among community members. Approaches such as micro-planning use peers to ensure more tailored services and help reach the most-at-risk and least served population members. Coupled with mapping and size estimates, the routine program monitoring data informed the pace of program infrastructure and services roll out as well as specified service utilization levels, which formed the basis for prioritization and setting benchmarks. Avahan's experiences and the emphasis on strengthening denominator thinking in prevention programming among high-risk populations has become a strong component of the current phase of the National AIDS Control Programme in India. Various methods for estimating the target population size have been reported from other countries; some of these were mapping and participatory methods similar to the approaches presented in the current review. Table 2 lists a few methods used in different countries. However, the different methods used for estimating the size of hidden populations in most countries suggest limited applicability of techniques such as capture-recapture and multiplier in routine program planning and implementation. Most of these methods are used in validating and evaluating the size estimates derived from routine program data or mapping and size estimation exercises. An evaluation of the Avahan program included cross-sectional surveys,${ }^{34}$ with one of the objectives being to provide independent estimates of the size of different highrisk populations. These external surveys were expected to provide data on size through different established indirect methods. ${ }^{5}$ The sizes from these estimations were similar to program estimates across different states and have been described and reported elsewhere..$^{35}$ Similar to the findings in this paper, the external surveys also suggest that mapping and size estimation processes improve with increased community involvement and program maturity. Poor availability of systematic documentation and reliance on information recall by key staff for mapping and size estimation are among the limitations of this review. Although mapping and size estimation exercises were carried out on a routine basis, results were not always documented. The data was often maintained in excel format and periodically revised to reflect findings. Despite this limitation, it is important to note that this paper describes field-level approaches for mapping and size estimation, on which there are few examples in existing literature. 
Table 2 Methods of estimating target population size from different countries\#

\begin{tabular}{|c|c|}
\hline Method used & Country \\
\hline Mapping and field surveys & Afghanistan ${ }^{25}$ and Ethiopia ${ }^{26}$ \\
\hline Participatory site assessment & Cambodia, Ecuador and India ${ }^{20}$ \\
\hline $\begin{array}{l}\text { Delphi technique and snowball } \\
\text { methods }\end{array}$ & West Bengal ${ }^{27}$ \\
\hline Capture-recapture method & $\begin{array}{l}\text { China, }{ }^{28} \text { Zimbabwe, }^{29} \text { Kenya }^{30} \text { EI } \\
\text { Salvador, }{ }^{31} \text { Bangladesh, }{ }^{32} \text { Madagascar } \\
\text { and India }\end{array}$ \\
\hline $\begin{array}{l}\text { Multiplier method used in } \\
\text { combination with census and } \\
\text { behavioral surveillance }\end{array}$ & China $^{33}$ \\
\hline
\end{tabular}

\section{Conclusion}

This review provides a description of the mapping and size estimation approaches used by Avahan: India AIDS Initiative, a large-scale HIV prevention program, to estimate the size of high-risk populations groups. Programmatically, mapping and size estimation exercises cannot be limited to 'just' counting the numbers; there is a need to collect and assimilate information on the risk behaviors, mobility of the target population and its vulnerability. Most approaches employed by Avahan attempted to address these issues. The overall experience shows that mapping and size estimation are important for initial resource allocation and strengthening program focus. Repeated mapping and size estimation along with program data improves ongoing assessment of program coverage and quality and informs effective program scale up. Incorporating routine size estimation activities into national programming is essential before the initiation of the next phase of the National AIDS Control Program in India and this will improve the programs ability to assess coverage and thereby identify gaps and inform effective scaling up over time.

\section{Acknowledgements}

The authors wish to thank the Avahan State Lead Partners in Maharashtra, Tamil Nadu, and Andhra Pradesh for their co-operation in gathering information on program mapping and size estimation. We also extend our appreciation to Dr. Niranjan Saggurti (Population Council), Mr. Virupax Ranebennur (FHI 360, Mumbai/India), Dr. Parmi Prabhakar (India HIV/AIDS Alliance, Hyderabad/India), Dr. Vijayaraman (Tamil Nadu AIDS Initiative), Ms. K. Dolly (HLFPPT), Dr. Sudipta Mondal (DFID), Dr. Shankar Talwar (independent consultant) for their inputs on the manuscript and Ms. Kavita Sharma (independent consultant) for editing.

\section{Conflict of interest}

Author declares that there is no conflict of interest.

\section{References}

1. Estimating the size of populations at risk for HIV: issues and methods UNAIDS/WHO Working Group on HIV/AIDS/STI Surveillance. 2003.

2. Kruse N, Behets FM, Vaovola G, et al. Participatory mapping of sex trade and enumeration of sex workers using capture-recapture methodology in Diego-Suarez, Madagascar. Sex Transm Dis. 2003;30(8):664-670.
3. Hook E, Regal R. Capture-recapture methods in epidemiology: methods and limitations. Epidemiol Rev. 1995;17(2):243-264.

4. Rongsheng L, Boheng L, Ping Y, et al. A Study on the Capture-Recapture Method for Estimating the Population Size of Injecting Drug Users in Southwest China. Journal of Health Science. 2005;51(4):405-409.

5. Vadivoo S, Gupte M, Adhikary R, et al. Appropriateness and execution challenges of three formal size estimation methods for high-risk populations in India. AIDS. 2008;22(Suppl 5):S137-S148.

6. Zhang D, Wang L, Lv F, et al. Advantages and challenges of using census and multiplier methods to estimate the number of female sex workers in a Chinese city. AIDS Care. 2007;19(1):17-19.

7. H Russell Bernard, Tim Hallett, Alexandrina Iovita, et al. Counting hardto-count populations: the network scale-up method for public health. Sexually Transmitted Infections. 2010;86(Suppl 2):ii11-ii15.

8. Estimation of the size of high risk groups and HIV prevalence in high risk groups in concentrated epidemics. UNAIDS, Amsterdam, Netherlands; 2008.

9. Vandepitte J, Lyerla R, Dallabetta G, et al. Estimates of the number of female sex workers in different regions of the world. Sex Transm Infect. 2006;82(Suppl 3):iii18-iii25.

10. Saidel T, Loo V, Salyuk T, et al. Applying Current Methods in Size Estimation for High Risk Groups in the Context of Concentrated Epidemics: Lessons Learned. JHASE. 2010:1-24.

11. Sentinel Surveillance data. Indian National AIDS Control Organization (NACO). India; 2003.

12. Avahan-The India AIDS Initiative: The Business of HIV Prevention at Scale. Bill \& Melinda Gates Foundation. India; 2008.

13. Avahan Common Minimum Program for HIV Prevention in India. Bill \& Melinda Gates Foundation. India; 2010.

14. Promising Approaches to Combination HIV Prevention Programming in Concentrated Epidemics. Bill \& Melinda Gates Foundation. India; 2011.

15. Bennett S, Singh S, Ozawa S, et al. Sustainability of donor programs: evaluating and informing the transition of a large HIV prevention program in India to local ownership. Glob Health Action. 2011;4.

16. Mapping of Select Population Groups Vulnerable to HIV/AIDSExperience during Preliminary Round of Field Work. International HIV/ AIDS Alliance. 2005.

17. Mapping of Key Population (Female Sex Workers (FSWs) and Men Having Sex with Men (MSM) Vulnerable to HIV/AIDS in 4 Districts of Andhra Pradesh Revised Methodology. International HIV/AIDS Alliance. 2005.

18. Targeted Interventions under NACP III: Operational Guidelines, (vol. 1), National AIDS Control Organisation (NACO) MoHFW. 2007.

19. Participatory site assessment- A guide to generating information to design community based interventions for HIV prevention programs in India. PATH. India; 2007.

20. Frontiers Prevention Project: Participatory Site Assessments in Cambodia, Ecuador and Andhra Pradesh State in India. International HIV/AIDS Alliance. India; 2003.

21. Kumar P. Consolidated report of size estimation methods across Avahan implementing partners, all six states (FSW, MSM and IDUs) [Unpublished manuscript]. 2010.

22. Process Documentation of Community Led Appraisal and Situational Study. HLFPPT; 2005.

23. Use It or Lose It: How Avahan Used Data to Shape Its HIV Prevention Efforts in India. Bill \& Melinda Gates Foundation, India; 2008. 
24. Managing HIV Prevention from the Ground Up: Avahan's Experience with Peer Led Outreach at Scale in India. Bill \& Melinda Gates Foundation, India; 2009.

25. Mapping and Situation Assessment of Key Populations at High Risk of HIV in Three Cities in Afghanistan: SAR AIDS Human Development Sector South Asia Region. The World Bank. 2008.

26. Mapping and census of female sex workers in ADDIS ABABA Ethiopia, FHI AACAHB. 2002.

27. Updating Mapping \& Size Estimation for Core Groups At Risk of HIV/ AIDS in West Bengal. West Bengal State AIDS Prevention and Control Society. India; 2007.

28. Luan R, Zeng G, Zhang D, et al. A study on methods of estimating the population size of men who have sex with men in Southwest China. Eur J Epidemiol. 2005;20(7):581-585.

29. Weir SS, Wilson D, Smith PJ, et al. Assessment of a Capture-Recapture Method for Estimating the Size of the Female Sex Worker Population in Bulawayo. Zimbabwe; 2003.

30. Vuylsteke B, Vandenhoudt H, Langat L, et al. Capture-recapture for estimating the size of the female sex worker population in three cities in Cote d'Ivoire and in Kisumu, western Kenya. Trop Med Int Health. 2010;15(12):1537-1543.
31. G P-B, Jo J, Me G, et al. How many men who have sex with men and female sex workers live in El Salvador? Using respondent-driven sampling and capture-recapture to estimate population sizes. Sex Transm Infect. 2011;87(4):279-282.

32. Si K, Bhuiya, Uddin AS. Application of the capture-recapture method for estimating number of mobile male sex workers in a port city of Bangladesh. J Health Popul Nutr. 2004;22(1):19-26.

33. Zhang D, Lv F, Wang L, et al. Estimating the population of female sex workers in two Chinese cities on the basis of the HIV/AIDS behavioural surveillance approach combined with a multiplier method. Sex Transm Infect. 2007;83(3):228-231.

34. Saidel T, Adhikary R, Mainkar M, et al. Baseline integrated behavioural and biological assessment among most at-risk populations in six highprevalence states of India: design and implementation challenges. AIDS. 2008;22(Suppl 5):S17-S34.

35. Gautam A, Adhikary R, Ramakrishnan L, et al. Changes in the sizes of key populations at high-risk for HIV: results from two rounds of biobehavioral surveys in three southern states of India. 2013. 